\title{
Flat band electrons and interactions in rhombohedral trilayer graphene
}

\author{
Hao Wang, ${ }^{1}$ Jin-Hua Gao, ${ }^{1,2}$ and Fu-Chun Zhang ${ }^{1,3}$ \\ ${ }^{1}$ Department of Physics, The University of Hong Kong, Hong Kong SAR, China \\ ${ }^{2}$ Department of Physics, Huazhong University of Science and Technology, Wuhan, China \\ ${ }^{3}$ Department of Physics, Zhejiang University, Hangzhou, 310027, China
}

(Received 6 November 2012; published 8 April 2013)

\begin{abstract}
Multilayer graphene systems with a rhombohedral stacking order harbor nearly flat bands in their single-particle spectrum. We propose ansatz states to describe the surface-localized states of flat band electrons. The absence of kinetic dispersion near the Fermi level leaves the interaction as a dominate mechanism to govern the low-energy physics of a low-density electron system. We build up an effective lattice model in two interacting low-energy bands, where the full terms of the Coulomb interaction, including those long-range and off-diagonal parts, have been considered. The interaction matrix coefficients in the many-body Hamiltonian model are directly calculated for a trilayer system using orthonormal Wannier basis. We then present a flat band projection to yield an interaction-only lattice model for flat band electrons. We find that this limited model might energetically favor a ferromagnetic quantum crystal under certain conditions.
\end{abstract}

DOI: $10.1103 /$ PhysRevB.87.155116

PACS number(s): 71.10.Fd, 71.27.+a, 73.21.Ac

\section{INTRODUCTION}

Graphene based structures have drawn numerous attentions due to their unique electronic properties. ${ }^{1,2}$ The rapid technique development enable people to engineer the graphene nanostructures in special designs, yielding rich band structure features. In recent years, great theoretical ${ }^{3-24}$ and experimental ${ }^{25-42}$ interests have been focused on the graphene multilayer systems. Different from the graphene monolayer, the band structure of the multilayer graphene system depends on its stacking order, i.e., the way of stacking the graphene sheets. Recently, the rhombohedral stacking multilayer graphene has drawn intensive research interests due to its intriguing band dispersion. It has two subbands near the neutral system Fermi level, one conduction band and one valence band with $|\epsilon| \sim k^{N}$ dispersion touching at $\epsilon=0$, where $N$ is the layer number. ${ }^{3}$ The rather flat energy bands near $\epsilon=0$ make the rhombohedral stacking multilayer graphene susceptible to the interaction. ${ }^{6}$ Thus the system is unstable towards quantum correlated phases, such as superconductors or ferromagnets. ${ }^{23,24}$

Some recent experiments ${ }^{41,42}$ in rhombohedral stacking graphene trilayer have shown hints of a gapped ground state, which is in sharp contrast with the gapless semiconducting ground state suggested in the noninteracting picture. Several symmetry-breaking correlated states, such as the layered antiferromagnetic, the quantum anomalous Hall, the quantum spin Hall, and the quantum valley Hall states, have been proposed as candidates for the gapped ground state., However, the theoretical predictions strongly depend on the model and parameters they chose. The detail properties of the ground state are still under debate.

Flat band electrons of the rhombohedral stacking graphene system are of particular interest, since it is believed that the correlated ground state results from the interplay between the electron-electron interaction and the peculiar flat energy bands near the Fermi level. For a low-density system, the dispersionless flat bands leave the Coulomb interaction predominantly to rule the low energy physics. This calls for a comprehensive evaluation of the effects from all interaction terms, including those long-range density-density repulsion terms and leading off-diagonal terms such as the direct spin exchange. The absence of the intraband screening in a flat band suggests that these nonlocal interactions would be relevant. Studies have shown that these nonlocal interactions can lead to exotic correlated phases, such as quantum crystal and quantum liquids..$^{43,44}$ In this paper, we theoretically investigate the flat band electrons and their interaction in the rhombohedral stacking graphene multilayer system. We establish a set of many-body Hamiltonian models that allow to appropriately include the effects from nonlocal interaction in addition to the Hubbard onsite term. Corresponding to the unique noninteracting band structure, a single-particle basis of Wannier functions is first constructed. We then use our basis to directly compute the matrix elements of a unscreened Coulomb interaction in two low-energy bands. A projection protocol has been presented to approach an approximate interaction-only lattice model in the flat band limit, which is highly nontrivial, incorporating two bands, long-range interactions, and spins. We argue that, at low densities, the long-range part of the interaction in this limit model might support ferromagnetic quantum crystals.

Our interaction model extends beyond the mean-field ${ }^{18}$ and renormalization group ${ }^{19,20}$ studies, where a screened interaction with either the onsite Hubbard term or a short-ranged interaction term is considered. Our study is also different from those with $a b$ initio calculations ${ }^{21}$ and Hartree-Fock approximations ${ }^{22}$ which rely on a certain local approximation to treat the nonlocal interaction and spin exchange terms. Alternatively, the Wannier basis allows us to directly calculate these nonlocal terms.

This paper is organized as follows. In Sec. II, we consider the band structure that arises from the noninteracting tightbinding model of rhombohedral stacking graphene systems. An ansatz wave function has been proposed to describe two flat bands. In Sec. III, we construct localized single-particle basis states, in the form of orthonormal Wannier functions, from carbon $\pi_{z}$ orbitals in graphene honeycomb lattices. Section IV uses the Wannier functions to explicitly compute 


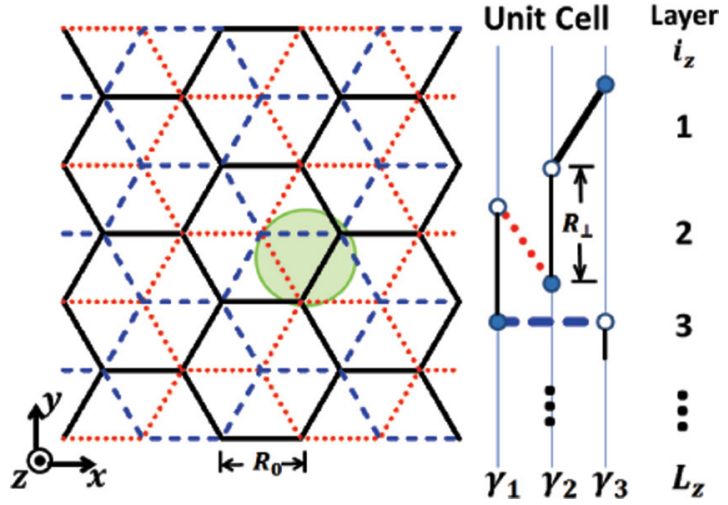

FIG. 1. (Color online) (Left) Schematic top-view of multiple layers of graphene sheets in the rhombohedral stacking order. Lines in solid, dot and dash types represent the in-plane carbon-carbon bonds at three neighboring layers, counted from top to bottom in the $\hat{z}$ direction. The shaded area corresponds to a single unit cell. (Right) Schematic side view of a unit cell in a triangular prism shape. The solid and open circles stand for the atomic sites of the sublattice $A$ and $B$, respectively. $\gamma_{i}$ are three corner axes of the prism.

the Coulomb interaction matrix elements for two low-energy bands. Section $\mathrm{V}$ defines a projection scheme that limits the total many-body model to the flat band portion of the single-particle spectrum and discusses the possible low-energy physics of this interaction-only lattice model. Section VI summarizes and looks forward to more accurate studies of the models constructed here.

\section{FLAT BANDS IN RHOMBOHEDRAL STACKING GRAPHENE SHEETS}

We consider interacting electrons hopping among carbon sites of rhombohedral graphene layers. In the left panel of Fig. 1, we schematically show the lattice of this stacking system. Two neighboring graphene layers have a relative in-plane shift along the carbon-carbon bond direction with the shift distance equal to the bond length $R_{0} \sim 1.42 \AA$. After three successive shifts, the forth layer recovers the same lattice as the first layer. We use $L_{z}$ to label the total number of stacking layers and the layer separation is similar as in graphite with $R_{\perp} \sim 3.35 \AA$. As shown in the right panel of Fig. 1, the primitive unit cell is in the shape of a triangular prism with the total number of atom sites $M=2 L_{z}$. Each layer of the unit cell contains two sublattice sites of $A$ and $B$ with perpendicular bonds to their counterpart sublattice site at the neighboring layers. The array of unit cells forms a two-dimensional Bravais triangular lattice with the lattice length $R_{c}=\sqrt{3} R_{0}$.

In a simple noninteracting picture, the minimum singleparticle tight-binding Hamiltonian is given as ${ }^{3}$

$$
H_{0}=-\sum_{\langle n, m\rangle}\left(t_{m n} \hat{c}_{n}^{\dagger} \hat{c}_{m}+\text { H.c. }\right),
$$

where the sum is along carbon-carbon bonds and the hopping integrals are taken $t_{\|}=3.16 \mathrm{eV}$ and $t_{\perp}=0.39 \mathrm{eV}$ for the intralayer and interlayer hoppings, respectively. ${ }^{45}$ The secondquantized operator $\hat{c}_{n}^{\dagger}$ creates a fermion at a site $n$. Labels $n$ and $m$ indicate lattice sites, in contrast to labels for unit cells, $i, j, k, l$, used in the following.

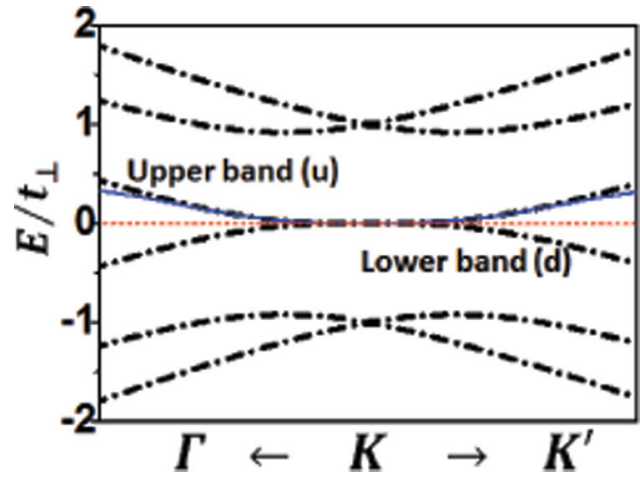

FIG. 2. (Color online) The dot-dashed lines indicate the energy eigenvalues of Eq. (1) vs wave vector for the rhombohedral graphene trilayer. The solid line shows the approximate expression for the energy [see Eq. (3)]. Two flat bands form near the valley points $K$ and $K^{\prime}$. In the large $L_{z}$ limit, the bands flatten.

Two bands near the Fermi level flatten around the corners ( $\mathbf{K}$ and $\mathbf{K}^{\prime}$ valley points) of the Brillouin zone (BZ). An example band structure for a trilayer system is shown in Fig. 2. Crossing the Fermi level, the conduction (upper band $u$ ) and valence (lower band $d$ ) bands are nearly degenerate with in-plane wave vectors $\mathbf{q}$ (relative to the valley points) in a region $|\mathbf{q}|<q^{\Delta}$ and form flat bands. For larger number of stacking layers, these bands can flatten considerably.

To model the two flat bands and examine the bandwidth, we construct analytical ansatz states in the linear combination of atom orbital basis as $\left(\phi_{A}, \phi_{B}\right)^{T}$ with $\phi_{A / B}=$ $\left(\phi_{A / B, i_{z}=1}(\mathbf{q}), \ldots, \phi_{A / B, i_{z}=L_{z}}(\mathbf{q})\right)$, where the sites of sublattice $A(B)$ on the bottom (top) layer have a direct link to the neighboring layer. The indices $i_{z}$ marked from 1 to $L_{z}$ represent the graphene layers from the topmost one to the bottom as shown in Fig. 1.

For a wave function to be exact for $E=0$, the mathematical necessary condition requires the wave function components between the neighboring layers to meet a certain relationship of

$$
\begin{aligned}
\frac{\phi_{A, i_{z}}(\mathbf{q})}{\phi_{A, i_{z}+1}(\mathbf{q})} & =\left(\frac{\phi_{B, i_{z}+1}(\mathbf{q})}{\phi_{B, i_{z}}(\mathbf{q})}\right)^{*}=p(\mathbf{q})^{-1}, \\
p(\mathbf{q}) & =-\frac{t_{\|}}{t_{\perp}}\left[e^{-i q_{x} R_{0}}+2 \cos \left(\frac{\sqrt{3}}{2} q_{y} R_{0}\right) e^{i q_{x} R_{0} / 2}\right] .
\end{aligned}
$$

Note that at the valley points of the $\mathbf{K}$ and $\mathbf{K}^{\prime}$, we have $|p(\mathbf{q})|=0$. The wave function is completely localized at two edge layers with the top layer occupied solely by the lattice $A$ and the bottom layer occupied solely by the lattice $B$. When the momentum is shifted away from the valley points, the wave function extends to the inner layers from the two edge layers. The ansatz wave functions in the vicinity of the valley points have the analytical form of $\Phi_{ \pm}(\mathbf{q})=\left(\phi_{A}, \pm \phi_{B}\right)^{T}$ with $\phi_{A}=$ $\left(1, p(\mathbf{q}), \ldots, p(\mathbf{q})^{L_{z}-1}\right)$ and $\phi_{B}=\left(\left(p^{*}(\mathbf{q})\right)^{L_{z}-1}, \ldots, p^{*}(\mathbf{q}), 1\right)$. In the general case with $\mid p(\mathbf{q} \mid \neq 1$, this ansatz state associates with a noneven occupation of the two sublattice sites on edge graphene layers.

Considering semi-infinite stacking layers of sublattices $A$ (edge at the top surface) and $B$ (edge at the bottom surface), the convergence of the wave function requires $|p(\mathbf{q})|<1$. This 
determines the valid range of a flat band ansatz wave function with a radius $q^{\Delta} /|\mathbf{K}| \approx\left(t_{\perp} / t_{\|}\right)(\sqrt{3} / 2 \pi)$ in the limit of $t_{\perp} / t_{\|}<$ 1. Here we see that an enhancement of the interlayer hopping leads to a larger flat band sector.

With the above ansatz states, the energy dispersion of bands $\Gamma=u, d$ in the flat band region can be computed explicitly:

$$
\begin{aligned}
\left|E_{\Gamma}(\mathbf{q})\right| & \approx \frac{\left|\Phi_{ \pm}(\mathbf{q})^{T} H_{0}(\mathbf{q}) \Phi_{ \pm}(\mathbf{q})\right|}{\left|\Phi_{ \pm}(\mathbf{q})\right|^{2}} \\
& =t_{\perp} \frac{\left|\operatorname{Re}\left[p(\mathbf{q})^{L_{z}}\right]\right|\left(1-|p(\mathbf{q})|^{2}\right)}{1-|p(\mathbf{q})|^{2 L_{z}}}
\end{aligned}
$$

with

$$
\begin{aligned}
H_{0}(\mathbf{q}) & =t_{\perp}\left(\begin{array}{cc}
0 & Q(\mathbf{q}) \\
Q^{\dagger}(\mathbf{q}) & 0
\end{array}\right), \\
Q(\mathbf{q}) & =\left(\begin{array}{cccc}
-p^{*}(\mathbf{q}) & 0 & . . & 0 \\
1 & -p^{*}(\mathbf{q}) & 0 & . . \\
: & : & : & : \\
0 & . . & 1 & -p^{*}(\mathbf{q})
\end{array}\right) .
\end{aligned}
$$

As shown in Fig. 2, the analytical dispersion agrees with those calculated directly from the tight-binding Hamiltonian in the vicinity of valley points, indicating that the ansatz wave function is an effective approximation to flat band states.

With Eq. (3), we can estimate the bandwidth of the two nearly flat bands using the energy value at the flat band boundary $\mathbf{q}^{\Delta}$. In the large $L_{z}$ limit, the bandwidth for states in the flat band sector vanishes as

$$
\left|E\left(\mathbf{q} \rightarrow \mathbf{q}^{\Delta}\right)\right| \rightarrow \frac{t_{\perp}}{L_{z}},
$$

indicating that the band dispersion plays a small role with increasing stacking number. Such a vanishing bandwidth leaves the interaction as the dominant term in the full manybody Hamiltonian of electrons.

For a dilute system with partially filled lattices, the lowerenergy physics of the electron system is mainly determined by the single-particle basis states within the flat band sectors near the Fermi level. Thus we project the Hamiltonian into the basis of flat band states in the approximation that $H_{0}$ adds an overall constant energy shift to the spectrum. Our Hamiltonian model becomes

$$
\begin{aligned}
H_{\text {total }} & =\sum_{\mathbf{q} \in \mathrm{BZ}, \sigma, \Gamma} E_{\Gamma}(\mathbf{q}) \hat{c}_{\mathbf{q} \sigma \Gamma}^{\dagger} \hat{c}_{\mathbf{q} \sigma \Gamma}+H_{V} \\
& \rightarrow \text { constant }+\mathcal{P}_{\mathrm{FB}}^{\dagger} H_{V} \mathcal{P}_{\mathrm{FB}},
\end{aligned}
$$

where the first equality is written in terms of the creation (annihilation) operator $\hat{c}_{\mathbf{q} \sigma \Gamma}^{\dagger}\left(\hat{c}_{\mathbf{q} \sigma \Gamma}\right)$ for a Bloch state at the wave vector $\mathbf{q}$ and band $\Gamma$, which is related to the operator for a single-particle basis state in the real space by the Fourier transform:

$$
\hat{c}_{j \sigma \Gamma}^{\dagger}=\frac{1}{\sqrt{N}} \sum_{\mathbf{q} \in \mathrm{BZ}} e^{i \mathbf{q} \cdot \mathbf{R}_{j}} \hat{c}_{\mathbf{q} \sigma \Gamma}^{\dagger} \cdot
$$

Here, $\mathbf{R}_{j}$ is the lattice vector of the $j$-th unit cell, $N$ denotes the number of unit cells in the system and $q$-space mesh in the $\mathrm{BZ}$, and $\sigma \in\{\uparrow, \downarrow\}$ labels spin. $\mathcal{P}_{\mathrm{FB}}^{\dagger}$ denotes a projection into flat bands such that the many-body eigenstates are constructed from Bloch states in the flat band sectors $|\mathbf{q}|<q^{\Delta}$.

To explore possible many-body ground states in the rhombohedral stacking graphene system, we need to construct an accurate form for Eq. (5) in the flat band basis. The absence of dispersion excludes intraband screening as in ordinary Fermi liquids. ${ }^{46}$ Thus many-body eigenstates are determined entirely by the interplay between various terms in the interaction. It is therefore crucial to accurately determine the interaction terms in Eq. (5) as prescribed by our choice of single-particle basis. In the next section, we describe how to construct orthonormal Wannier functions to serve as single-particle basis states.

\section{SINGLE-PARTICLE BASIS STATES: LOW-ENERGY BAND WANNIER FUNCTIONS}

In this section, we superpose overlapping carbon $\pi_{z}$ orbitals to form orthogonal Wannier functions. The Wannier functions will then be used to accurately determine interaction matrix elements in later sections.

In an isolated band the Wannier functions are given by

$$
W_{j}(\mathbf{r})=W_{0}\left(\mathbf{r}-\mathbf{R}_{j}\right)=\frac{1}{N} \sum_{\mathbf{q}} e^{-i \mathbf{q} \cdot \mathbf{R}_{j}} \Psi_{\mathbf{q}}(\mathbf{r}),
$$

where momenta q sum over $N$ discrete values inside the entire BZ. The Bloch functions are $\Psi_{\mathbf{q}}(\mathbf{r})=\sum_{m=1}^{M} C_{m \mathbf{q}} \chi_{m \mathbf{q}}(\mathbf{r})$.

To make the contact with first-principles calculations ${ }^{21}$ we form Bloch functions from carbon $\pi_{z}$ orbitals, $\phi(\mathbf{r})=\sqrt{\xi^{5} / \pi} z e^{-\xi r}$. The basis states become $\chi_{m \mathbf{q}}(\mathbf{r})=$ $(1 / \sqrt{N}) \sum_{\mathbf{q}} e^{i \mathbf{q} \cdot \mathbf{R}_{j}} \phi\left(\mathbf{r}-\mathbf{r}_{m j}\right)$, where $\mathbf{r}_{m j}=\mathbf{R}_{j}+\mathbf{T}_{m}$ is the location of the $m$ th atom in the $j$ th unit cell.

The coefficients $C_{m \mathbf{q}}$ and energy eigenvalues $E(\mathbf{q})$ are obtained from diagonalization of the secular equation:

$$
\left[\tilde{O}^{-1} \tilde{H}(\mathbf{q})\right] \mathbf{C}_{\mathbf{q}}=E(\mathbf{q}) \mathbf{C}_{\mathbf{q}},
$$

where the matrix $\tilde{H}$ follows from the tight-binding Hamiltonian $H_{0}$ with elements $\tilde{H}(\mathbf{q})_{m n}=\int d \mathbf{r} \chi_{m \mathbf{q}}^{*}(\mathbf{r}) H_{0} \chi_{n \mathbf{q}}(\mathbf{r})$. The overlap matrix $\tilde{O}$ is taken as the identity matrix in the tight-binding approximation. The eigenvectors $\mathbf{C}_{\mathbf{q}} \equiv$ $\left\{C_{1 \mathbf{q}}, \ldots, C_{M \mathbf{q}}\right\}^{T}$ yield the coefficients used in the definition of the Wannier functions.

To construct orthonormal Wannier function, a specific set of single-particle basis states are chosen by enforcing $C_{m \mathbf{q}}$ at the edge atomic sites $m=1$ and $m=M$ conjugate. The resulting Wannier functions $W_{j}(\mathbf{r})$ are real and localized at $\mathbf{R}_{j}$ with the certain symmetry between top and bottom portions in the stacking direction $\hat{z}$.

We can write the Wannier function at the origin as a summation over all local atomic orbitals $\phi(\mathbf{r})$, i.e.,

$$
W_{0}(\mathbf{r})=N_{f} \sum_{m=1}^{M} \sum_{i=0}^{N-1} \alpha_{m i} \phi\left(\mathbf{r}-\mathbf{r}_{m i}\right)
$$

with weights $\alpha_{m j}=\sum_{\mathbf{q}} C_{m \mathbf{q}} e^{i \mathbf{q} \cdot \mathbf{R}_{j}}$ and the normalization constant $N_{f}$. A denser sampling in momentum space (i.e., larger $N$ ) yields more accurate Wannier functions. In practice, we find that the Wannier function has already converged when taking $N=1261$ for $L_{z}=3$. 

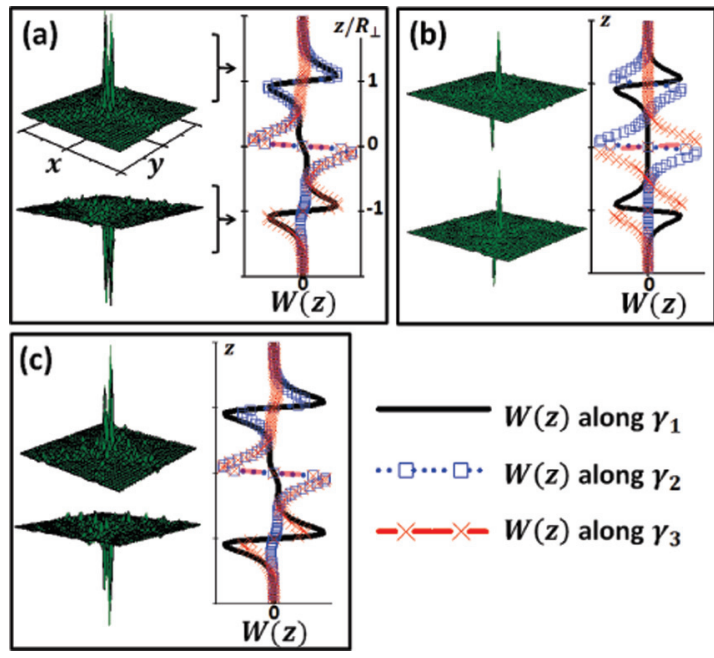

FIG. 3. (Color online) Wannier functions of trilayer graphene sheets. (a) $u$-band case: Two three-dimensional plots on the left represent the distribution of the Wannier functions in the $x y$ plane at the $z$ positions right above the top layer and right below the bottom layer, respectively. The twin peaks locate at two sublattice sites of the edge layer in the original unit cell. The cartoon on the right plots the distribution of the Wannier function in the $\hat{z}$ direction along three corner axes of the original unit cell. (b) The same plots as (a) but for the $d$-band case. (c) $u$-band Wannier function with adjusted parameters $t_{\perp}=t_{\|}$to emphasize the flat band effect.

We can extend our construction of the Wannier functions to include both the upper and lower bands. The Wannier functions of these two low-energy bands in a trilayer system are shown in Fig. 3. We note that these two Wannier functions mainly localize at the original unit cell with the reflection symmetry (antisymmetry) along a center line $(\sqrt{3}, 1,0)$ for the upper (lower) band, decaying rapidly within several cell lengths. In the plots of Wannier functions as a function of $z$ positions as shown in Figs. 3(a) and 3(b), the Wannier functions are mostly distributed in a narrow region around each layer with the node on the layer. This is due to the property of underlying $\pi_{z}$ orbitals. We note that there exists a large portion of the Wannier function around the middle layer, indicating the contribution from those extensive states with momenta outside the flat band region. Under the given hopping parameters of $t_{\perp} / t_{\|} \sim 0.1$, the two sublattices near evenly occupy each layer.

The Wannier functions built here integrate over the entire BZ. Thus the extensive states from the large nonflat region may shield the real feature of the surface-localized state in the flat band sectors. Based on the analysis in the previous section, we have learned that the size of the flat band region and the flatness of the bands are proportional to the hopping parameter $t_{\perp}$. To explore the effect from the relevant flat band states in the Wannier functions, we study the case with the exaggerative parameter $t_{\perp}=t_{\|}$. As shown in Fig. 3(c), the nonbalanced occupation between sublattices $A$ and $B$ at two surface layers magnifies as the flat band region expands, consistent with the property of the ansatz flat band state in the previous section. Meanwhile, the relative portion of the extensive Wannier function around the middle layer also reduces as expected. The flat band induced asymmetric occupation of two sublattices in the surface layers may justify the origin of the gapped symmetry-breaking states proposed by earlier theoretical studies. $6,19,22$

\section{COULOMB INTERACTION MODEL}

For a dilute system where the chemical potential lies between the two nearly flat bands, the Coulomb interaction can in principle favor occupancy of both bands or a single band. As a first approximation, we assume that the valence band is inert and only the conduction band $u$ is active.

An unscreened Coulomb interaction in a single band has a second-quantized many-body form of

$$
\sum_{i, j, k, l ; \sigma, \sigma^{\prime}} \mathcal{V}_{i j k l} \hat{c}_{i \sigma}^{\dagger} \hat{c}_{j \sigma^{\prime}}^{\dagger} \hat{c}_{k \sigma^{\prime}} \hat{c}_{l \sigma}
$$

where the operators $\hat{c}_{i \sigma}^{\dagger}\left(\hat{c}_{i \sigma}\right)$ create (annihilate) a fermion with spin $\sigma$ in a Wannier state centered at the $i$ th unit cell. The matrix elements $\mathcal{V}$ are determined by the Wannier basis given in the last section. We can rewrite the above many-body Coulomb interaction in a suggestive form:

$$
\begin{aligned}
H_{V}^{u} & =V_{0} \sum_{i} n_{i \uparrow} n_{i \downarrow}+\sum_{i<j} V_{i j} n_{i} n_{j}-\sum_{i<j} J_{i j} \mathbf{S}_{i} \cdot \mathbf{S}_{j} \\
& +\frac{1}{2} \sum_{\{i, j\} \nsubseteq\{k, l\} ; \sigma, \sigma^{\prime}} V_{i j k l} \hat{c}_{i \sigma}^{\dagger} \hat{c}_{j \sigma^{\prime}}^{\dagger} \hat{c}_{k \sigma^{\prime}} \hat{c}_{l \sigma} .
\end{aligned}
$$

Here, the single-component and total density operators are $n_{i \sigma}=\hat{c}_{i \sigma}^{\dagger} \hat{c}_{i \sigma}$ and $n_{i}=n_{i \uparrow}+n_{i \downarrow}$, respectively. The spin operators $\mathbf{S}_{i}=(1 / 2) \sum_{\sigma \sigma^{\prime}} \hat{c}_{i \sigma}^{\dagger} \tilde{\boldsymbol{\sigma}}_{\sigma \sigma^{\prime}} \hat{c}_{i \sigma^{\prime}}$ are defined in terms of the Pauli matrices $\tilde{\boldsymbol{\sigma}}$.

Equation (11) keeps all terms in the full Coulomb interaction. The first term is the ordinary onsite Hubbard term used in some mean-field studies of multilayer graphene systems. ${ }^{17,18}$ The second term captures the diagonal portion of the Coulomb interaction at long range, which favors certain charge order, such as a two-dimensional Wigner crystal. The absence of a dispersion in a low-density system implies that this term can be relevant and must be kept in accurate models. The third term, the direct exchange term, favors ferromagnetism for $J_{i j}>0$. The last term represents the remaining off-diagonal terms due to the Coulomb interaction, which are much small compared to the first three terms for a single band according to our direct calculation.

The matrix elements in Eq. (11) can be computed explicitly using the Wannier basis in the $u$ band, as shown in Appendix, see Eq. (A1). To calculate these integral equations, we have approximated the exponential part of the $\pi_{z}$ orbital as a linear combination of three Gaussian functions: $\phi(\mathbf{r}) \approx$ $\sum_{s} \gamma_{s}\left(128 \beta_{s}^{5} / \pi^{3}\right)^{1 / 4} z e^{-\beta_{s} r^{2}}$, where the parameters $\gamma_{s}$ and $\beta_{s}$ are obtained from the STO-3G package. ${ }^{47}$ Data for fitting the $\pi_{z}$ orbital with $\xi=1.72$ are listed in Table I. For the numerical

TABLE I. Fitting parameters for the Gaussian approximation to the $\pi_{z}$ orbital with $\xi=1.72$.

\begin{tabular}{llll}
\hline \hline$s$ & \multicolumn{1}{c}{1} & \multicolumn{1}{c}{2} & \multicolumn{1}{c}{3} \\
\hline$\gamma_{s}$ & 0.15591627 & 0.60768372 & 0.39195739 \\
$\beta_{s}$ & 2.9412494 & 0.6834831 & 0.2222899 \\
\hline \hline
\end{tabular}


TABLE II. Matrix elements for one-band ( $u$ band) case of the $L_{z}=3$ system with unit cell separations of up to $3 R_{c}$.

\begin{tabular}{lccccc}
\hline \hline$V_{0}=3.587 \times 10^{-1}$ & & & & \\
\hline $\mathbf{R}_{i}-\mathbf{R}_{j} \mid / R_{c}$ & 1 & $\sqrt{3}$ & 2 & $\sqrt{7}$ & 3 \\
$J_{i j}$ & $2.136 \times 10^{-3}$ & $2.232 \times 10^{-3}$ & $9.703 \times 10^{-4}$ & $5.273 \times 10^{-4}$ & $6.075 \times 10^{-5}$ \\
$V_{i j}$ & $2.007 \times 10^{-1}$ & $1.462 \times 10^{-1}$ & $1.319 \times 10^{-1}$ & $1.080 \times 10^{-1}$ & $9.751 \times 10^{-2}$ \\
\hline \hline
\end{tabular}

results shown here and in the following tables, we use the Bohr radius, $a_{0}=0.53 \AA$, as the unit length and the Coulomb energy $e^{2} / 4 \pi \epsilon a_{0}(\sim 27.2 \mathrm{eV}$ in vacuum $)$ as the unit of energy.

Table II lists the coefficients computed for a trilayer system. As we see, all coefficients are positive and can be sorted by $V_{0}>V_{i j}>J_{i j}>0$. These coefficients suggest that a partially filled single band supports the formation of ferromagnetic crystals.

However, the large Coulomb interaction may cause mixing between the $u$ and $d$ bands. We need consider a more comprehensive two-band interaction model with Wannier functions in both the $u$ and $d$ bands. The interaction Hamiltonian is dominated by the following terms:

$$
\begin{aligned}
H_{V}^{u d}= & \sum_{i, \Gamma} V_{0}^{\Gamma} n_{i \Gamma \uparrow} n_{i \Gamma \downarrow}+\sum_{i, \sigma, \Gamma \neq \Gamma^{\prime}} V_{0}^{\prime} n_{i \Gamma \sigma} n_{i \Gamma^{\prime} \sigma} \\
& +\sum_{i}\left(\sum_{\Gamma \neq \Gamma^{\prime}} V_{i i}^{\prime} n_{i \Gamma} n_{i \Gamma^{\prime}}-J_{i i}^{\prime} \mathbf{S}_{i u} \cdot \mathbf{S}_{i d}\right) \\
& +\sum_{i<j, \Gamma}\left(V_{i j}^{\Gamma} n_{i \Gamma} n_{j \Gamma}-J_{i j}^{\Gamma} \mathbf{S}_{i \Gamma} \cdot \mathbf{S}_{j \Gamma}\right) \\
& +\sum_{i<j} \sum_{\Gamma \neq \Gamma^{\prime}}\left(V_{i j}^{\prime} n_{i \Gamma} n_{j \Gamma^{\prime}}-J_{i j}^{\prime} \mathbf{S}_{i \Gamma} \cdot \mathbf{S}_{j \Gamma^{\prime}}\right) \\
& +\sum_{i<j} \sum_{\Gamma \neq \Gamma^{\prime}} \sum_{\sigma, \sigma^{\prime}}\left(V_{i j}^{\prime \prime} \hat{c}_{i \Gamma \sigma}^{\dagger} \hat{c}_{j \Gamma^{\prime} \sigma^{\prime}}^{\dagger} \hat{c}_{j \Gamma \sigma^{\prime}} \hat{c}_{i \Gamma^{\prime} \sigma}\right. \\
& \left.+V_{i j}^{\prime \prime \prime} \hat{c}_{i \Gamma \sigma}^{\dagger} \hat{c}_{j \Gamma^{\prime} \sigma^{\prime}}^{\dagger} \hat{c}_{i \Gamma^{\prime} \sigma^{\prime}} \hat{c}_{j \Gamma \sigma}\right) .
\end{aligned}
$$

We have checked, by direct calculations, that other terms involving three or four centers are much smaller than terms kept here. In Eq. (12), we see the Hubbard and ferromagnetic terms as in the one-band analysis. Besides, we have the nontrivial band exchange as the last term. The integral equations for all coefficients in Eq. (12) are listed in Appendix.

Table III shows numerically computed coefficients for the two-band model in a trilayer system. Rows 1-3 exhibit several leading terms of the diagonal components of Coulomb interaction, which primarily determine the charge degrees of freedom. Rows 4-6 govern the spin degrees of freedom. The positive elements support ferromagnetism. The last two rows give rise to band exchange effects.

The calculated coefficients of the onsite Coulomb repulsion have values of 2-5 eV with an estimated effective dielectric constant $\epsilon=2$ in graphene systems, which are consistent with the parameter range in a mean-field analysis ${ }^{18}$ for the experimentally observed energy gap. ${ }^{41,42}$ We also note that the long-range interaction terms of up to the fifth nearest neighbors (rows 1-3) have a magnitude comparable to the on-site terms, indicating that the effective interaction range could be much longer than the usual screened interaction treatments with up to nearest or next-to-nearest neighbors. Based on the energetic argument, these long-range terms are relevant and should be included to discuss the possible low-energy states of a dilute system.

\section{FLAT-BAND PROJECTION}

In this section, we construct a set of operators that allow flat band projection of the many-body Hamiltonian model constructed in the previous sections. We then discuss the possible low-energy physics under certain conditions.

To enforce flat band projection, we limit all $q$-space sums to the flat band region (FBR) $|\mathbf{q}|<q^{\Delta}$. We first consider a state operator in a single band that limits itself to the FBR:

$$
\hat{b}_{j \sigma}^{\dagger} \equiv \frac{1}{N} \sum_{l} \sum_{\mathbf{q} \in \mathrm{FBR}} e^{i \mathbf{q} \cdot\left(\mathbf{R}_{j}-\mathbf{R}_{l}\right)} \hat{c}_{l \sigma}^{\dagger} .
$$

This operator creates states centered around the unit cell at $\mathbf{R}_{j}$ while can overlap considerably with neighbors, indicating that the projection into a flat band delocalizes basis states. In the limit that the flat band encompasses the entire Brillouin zone, we have $\hat{b}_{j \sigma}^{\dagger} \rightarrow \hat{c}_{j \sigma}^{\dagger}$

We can then rewrite our model in terms of projected density and spin operators. The single-component and total projected density operators are $\rho_{i \sigma} \equiv \hat{b}_{i \sigma}^{\dagger} \hat{b}_{i \sigma}$ and $\rho_{i} \equiv \rho_{i \uparrow}+$ $\rho_{i \downarrow}$, respectively. The projected spin operators are defined as

$$
\boldsymbol{\oiint}_{j} \equiv \frac{1}{2 N} \sum_{\sigma \sigma^{\prime}} \sum_{\mathbf{q}, \mathbf{q}^{\prime} \in \mathrm{FBR}} e^{i\left(\mathbf{q}-\mathbf{q}^{\prime}\right) \cdot \mathbf{R}_{j}} \hat{c}_{\mathbf{q} \sigma}^{\dagger} \tilde{\boldsymbol{\sigma}}_{\sigma \sigma^{\prime}} \hat{c}_{\mathbf{q}^{\prime} \sigma^{\prime}}
$$

Note that these projected operators do not exhibit ordinary commutation relations because the underlying basis states are delocalized.

TABLE III. Same as the Table II but for the two-band case.

\begin{tabular}{lclc}
\hline \hline$V_{0}^{d}=1.495 \times 10^{-1}$ & & \\
$V_{i i}^{\prime}=2.192 \times 10^{-1}$ & & $V_{0}^{u}=3.587 \times 10^{-1}$ & \\
$J_{i i}^{\prime}=9.864 \times 10^{-2}$ & & $V_{0}^{\prime}=4.932 \times 10^{-2}$ & \\
\hline$\left|\mathbf{R}_{i}-\mathbf{R}_{j}\right| / R_{c}$ & 1 & 2 & 3 \\
$V_{i j}^{d}$ & $8.419 \times 10^{-2}$ & $5.527 \times 10^{-2}$ & $4.079 \times 10^{-2}$ \\
$V_{i j}^{u}$ & $2.007 \times 10^{-1}$ & $1.319 \times 10^{-1}$ & $9.751 \times 10^{-2}$ \\
$V_{i j}^{\prime}$ & $1.304 \times 10^{-1}$ & $8.562 \times 10^{-2}$ & $6.313 \times 10^{-2}$ \\
$J_{i j}^{d}$ & $8.877 \times 10^{-4}$ & $3.965 \times 10^{-4}$ & $2.443 \times 10^{-5}$ \\
$J_{i j}^{u}$ & $2.136 \times 10^{-3}$ & $9.703 \times 10^{-4}$ & $6.075 \times 10^{-5}$ \\
$J_{i j}^{\prime}$ & $2.726 \times 10^{-4}$ & $2.052 \times 10^{-4}$ & $2.058 \times 10^{-5}$ \\
$V_{i j}^{\prime \prime}$ & $7.467 \times 10^{-4}$ & $7.920 \times 10^{-5}$ & $2.994 \times 10^{-5}$ \\
$V_{i j}^{\prime \prime \prime}$ & $6.885 \times 10^{-4}$ & $3.100 \times 10^{-4}$ & $3.853 \times 10^{-5}$ \\
\hline \hline
\end{tabular}


The projected Hamiltonian can be rewritten entirely in terms of the above projected operators. Starting from an unprojected interaction model, we impose projection using the following replacements: $c \rightarrow b, n \rightarrow \rho$, and $\mathbf{S} \rightarrow \mathbf{S}$. Considering the intrinsic energetic ordering as shown in Table III, we rewrite the two-band interaction Hamiltonian in the projected space:

$$
\begin{aligned}
\mathcal{P}_{\mathrm{FB}}^{\dagger} H_{V}^{u d} \mathcal{P}_{\mathrm{FB}}= & \sum_{i, \Gamma} V_{0}^{\Gamma} \rho_{i \Gamma \uparrow} \rho_{i \Gamma \downarrow}+\sum_{i, \sigma, \Gamma \neq \Gamma^{\prime}} V_{0}^{\prime} \rho_{i \Gamma \sigma} \rho_{i \Gamma^{\prime} \sigma} \\
& +\sum_{i, j, \Gamma, \Gamma^{\prime}}\left(\bar{V}_{i j}^{\Gamma, \Gamma^{\prime}} \rho_{i \Gamma} \rho_{j \Gamma^{\prime}}-\bar{J}_{i j}^{\Gamma, \Gamma^{\prime}} \boldsymbol{\$}_{i \Gamma} \cdot \boldsymbol{\$}_{j \Gamma^{\prime}}\right) \\
& +H_{\text {Band-exch }},
\end{aligned}
$$

where we have redefined the diagonal Coulomb terms: $\bar{V}_{i<j}^{\Gamma \neq \Gamma^{\prime}} \equiv V_{i j}^{\prime}, \bar{V}_{i i}^{\Gamma=d, \Gamma^{\prime}=u} \equiv V_{i i}^{\prime}$, and $\bar{V}_{i<j}^{\Gamma=\Gamma^{\prime}} \equiv V_{i j}^{\Gamma}$, otherwise $\bar{V}_{i j}^{\Gamma, \Gamma^{\prime}}=0$. We have also redefined the off-diagonal exchange terms: $\bar{J}_{i<j}^{\Gamma \neq \Gamma^{\prime}} \equiv J_{i j}^{\prime}, \bar{J}_{i i}^{\Gamma=d, \Gamma^{\prime}=u} \equiv J_{i i}^{\prime}$, and $\bar{J}_{i<j}^{\Gamma=\Gamma^{\prime}} \equiv J_{i j}^{\Gamma}$, otherwise $\bar{J}_{i j}^{\Gamma, \Gamma^{\prime}}=0$. The last term in Eq. (15) corresponds to the last term in Eq. (12).

Let us consider a conditional dilute system with the Fermi energy away from the charge neutrality point, where the band far away from Fermi level is approximately inert and the band-exchange terms can be ignored. The first three terms in Eq. (15) will impose a rigid charge order analogy to the classical Wigner crystal. However, here the charges are significantly delocalized. A finite overlap among neighbors implies that the charges exist in a superposition of several different unit cells at once, indicating a quantum crystal. The forth term corresponds to an effective Heisenberg model that favors ferromagnetism between neighboring cell spins. Thus the projected system favors a ground state as in ferromagnetic quantum crystals. Correspondingly, low-energy spin excitations could be ferromagnetic magnons. ${ }^{44}$ At low temperature, the ferromagnetic order among two-dimensional cell spins could be detectable using a magnetic scanning probe microscopy technique, such as magnetic force microscopy and spin-polarized scanning tunneling microscopy. ${ }^{48}$ We note that this in-plane ferromagnetic order is also suggested by several theoretical models ${ }^{18-22}$ in the distinct system where the Fermi level is near the charge neutrality point and the correlation between both flat bands is involved.

\section{SUMMARY AND OUTLOOK}

We construct an interaction lattice model for flat band electrons in rhombohedral stacking graphene layers. An ansatz wave function has been proposed to describe the properties of flat band states emerging in the single-particle spectrum of the system. A single-particle basis of orthonormal Wannier functions was built from the carbon $\pi_{z}$ orbitals of the underlying graphene honeycomb lattice. We use this single-particle basis to explicitly compute the Coulomb matrix elements. The total model was then projected onto the flat bands, suggesting a ferromagnetic quantum crystal ground state under certain assumptions.

Numerical results are shown here for the trilayer system. However, the formulas of our model and approach are general to the rhombohedral graphene multilayer system. In a separate calculation with more layers, we have found a similar feature of the Wannier basis and a similar relative order among interaction coefficients. Our flat band model sets the stage for more accurate studies with a combination of variational studies and diagonalization to verify possible ground and excited states. ${ }^{43}$

We also want to stress the difference between the work presented here and a previous mean-field study. ${ }^{18}$ Our interaction model includes a full consideration of the nonlocal interaction terms from two low-energy bands. The mean-field study ${ }^{18}$ takes interaction contribution from all bands but only counts the on-site interaction term. Our model can be applicable in the limit case with a large stacking number and weak interaction. There, the low-energy properties of the system are most relevant to two extremely flat band portions. In another case where the interaction is strong and the screening effect from those dispersive higher-energy bands is not negligible, the mean-field treatment would be justified.

The constructed model focuses on key physics of interacting flat bands but excludes a number of realistic effects. For example, longer-range hopping can cause the trigonal warping effect and other effects, which distort the flat bands. The experimental conditions, such as defects and substrate disorder can also destroy the flat band approximation. We apply the flat band limit considering that these deformations are less than the estimated bandwidth in Eq. (4). In addition, interband screening from nearby bands could lead to the corrections to the Coulomb interaction studied here.

\section{ACKNOWLEDGMENTS}

We acknowledge part of financial support from HKSAR RGC grant HKU 701010. JHG is supported by National Natural Science Foundation of China (Project No. 11274129).

\section{APPENDIX}

The coefficients in Eqs. (11) and (12) are given by

$$
\begin{aligned}
V_{0}^{\Gamma} & =\int \frac{d^{3} \mathbf{r} d^{3} \mathbf{r}^{\prime}}{\left|\mathbf{r}-\mathbf{r}^{\prime}\right|}\left|W_{0 \Gamma}(\mathbf{r}) W_{0 \Gamma}\left(\mathbf{r}^{\prime}\right)\right|^{2}, \\
J_{i j}^{\Gamma} & =2 \int \frac{d^{3} \mathbf{r} d^{3} \mathbf{r}^{\prime}}{\left|\mathbf{r}-\mathbf{r}^{\prime}\right|} W_{i \Gamma}^{*}(\mathbf{r}) W_{j \Gamma}(\mathbf{r}) W_{i \Gamma}\left(\mathbf{r}^{\prime}\right) W_{j \Gamma}^{*}\left(\mathbf{r}^{\prime}\right), \\
V_{i j}^{\Gamma} & =\int \frac{d^{3} \mathbf{r} d^{3} \mathbf{r}^{\prime}}{\left|\mathbf{r}-\mathbf{r}^{\prime}\right|}\left|W_{i \Gamma}(\mathbf{r}) W_{j \Gamma}\left(\mathbf{r}^{\prime}\right)\right|^{2}-\frac{1}{4} J_{i j}^{\Gamma}, \\
J_{i j}^{\prime} & =2 \int \frac{d^{3} \mathbf{r} d^{3} \mathbf{r}^{\prime}}{\left|\mathbf{r}-\mathbf{r}^{\prime}\right|} W_{i u}^{*}(\mathbf{r}) W_{j d}(\mathbf{r}) W_{i u}\left(\mathbf{r}^{\prime}\right) W_{j d}^{*}\left(\mathbf{r}^{\prime}\right), \\
V_{i j}^{\prime} & =\int \frac{d^{3} \mathbf{r} d^{3} \mathbf{r}^{\prime}}{\left|\mathbf{r}-\mathbf{r}^{\prime}\right|}\left|W_{i u}(\mathbf{r}) W_{j d}\left(\mathbf{r}^{\prime}\right)\right|^{2}-\frac{1}{4} J_{i j}^{\prime}, \quad V_{0}^{\prime}=\frac{1}{2} J_{i i}^{\prime}, \\
V_{i j}^{\prime \prime} & =\int \frac{d^{3} \mathbf{r} d^{3} \mathbf{r}^{\prime}}{\left|\mathbf{r}-\mathbf{r}^{\prime}\right|} W_{i u}^{*}(\mathbf{r}) W_{i d}(\mathbf{r}) W_{j u}\left(\mathbf{r}^{\prime}\right) W_{j d}^{*}\left(\mathbf{r}^{\prime}\right), \\
V_{i j}^{\prime \prime \prime} & =\int \frac{d^{3} \mathbf{r} d^{3} \mathbf{r}^{\prime}}{\left|\mathbf{r}-\mathbf{r}^{\prime}\right|} W_{i u}^{*}(\mathbf{r}) W_{j u}(\mathbf{r}) W_{i d}\left(\mathbf{r}^{\prime}\right) W_{j d}^{*}\left(\mathbf{r}^{\prime}\right), \\
V_{i j k l} & =\int \frac{d^{3} \mathbf{r} d^{3} \mathbf{r}^{\prime}}{\left|\mathbf{r}-\mathbf{r}^{\prime}\right|} W_{i u}^{*}(\mathbf{r}) W_{l u}(\mathbf{r}) W_{j u}^{*}\left(\mathbf{r}^{\prime}\right) W_{k u}\left(\mathbf{r}^{\prime}\right) .
\end{aligned}
$$

The last term is used only in Eq. (11). 
${ }^{1}$ K. S. Novoselov, A. K. Geim, S. V. Morozov, D. Jiang, Y. Zhang, S. V. Dubonos, I. V. Grigorieva, and A. A. Firsov, Science 306, 666 (2004).

${ }^{2}$ A. H. Castro Neto, F. Guinea, N. M. R. Peres, K. S. Novoselov, and A. K. Geim, Rev. Mod. Phys. 81, 109 (2009).

${ }^{3}$ F. Guinea, A. H. Castro Neto, and N. M. R. Peres, Phys. Rev. B 73, 245426 (2006).

${ }^{4}$ S. Latil and L. Henrard, Phys. Rev. Lett. 97, 036803 (2006).

${ }^{5}$ H. Min and A. H. MacDonald, Phys. Rev. B 77, 155416 (2008).

${ }^{6}$ F. Zhang, B. Sahu, H. Min, and A. H. MacDonald, Phys. Rev. B 82, 035409 (2010); F. Zhang, J. Jung, G. A. Fiete, Q. Niu, and A. H. MacDonald, Phys. Rev. Lett. 106, 156801 (2011); Fan Zhang, Dagim Tilahun, and A. H. MacDonald, Phys. Rev. B 85, 165139 (2012).

${ }^{7}$ M. Koshino and E. McCann, Phys. Rev. B 83, 165443 (2011).

${ }^{8}$ Sh. Yuan, R. Roldan, and M. I. Katsnelson, Phys. Rev. B 84, 125455 (2011).

${ }^{9}$ R. van Gelderen, L.-K. Lim, and C. M. Smith, Phys. Rev. B 84, 155446 (2011).

${ }^{10}$ T. Wakutsu, M. Nakamura, and B. Dóra, Phys. Rev. B 85, 033403 (2012).

${ }^{11}$ A. A. Avetisyan, B. Partoens, and F. M. Peeters, Phys. Rev. B 80, 195401 (2009).

${ }^{12}$ M. Koshino and E. McCann, Phys. Rev. B 80, 165409 (2009); M. Koshino, ibid. 81, 125304 (2010).

${ }^{13}$ S. B. Kumar and J. Guo, Appl. Phys. Lett. 98, 222101 (2011); 100, 163102 (2012).

${ }^{14}$ M. Otani and Y. Takagi, M. Koshino and S. Okada, Appl. Phys. Lett. 96, 242504 (2010).

${ }^{15}$ J.-A. Yan, W. Y. Ruan, and M. Y. Chou, Phys. Rev. B 83, 245418 (2011).

${ }^{16}$ M. M. Scherer, S. Uebelacker, and C. Honerkamp, Phys. Rev. B 85, 235408 (2012).

${ }^{17}$ T. C. Lang, Z. Y. Meng, M. M. Scherer, S. Uebelacker, F. F. Assaad, A. Muramatsu, C. Honerkamp, and S. Wessel, Phys. Rev. Lett. 109, 126402 (2012).

${ }^{18}$ D.-H. Xu, J. Yuan, Z.-J. Yao, Y. Zhou, J.-H. Gao, and F.-C. Zhang, Phys. Rev. B 86, 201404(R) (2012).

${ }^{19}$ M. M. Scherer, S. Uebelacker, D. D. Scherer, and C. Honerkamp, Phys. Rev. B 86, 155415 (2012).

${ }^{20}$ V. Cvetkovic and O. Vafek, arXiv:1210.4923.

${ }^{21}$ M. Otani, M. Koshino, Y. Takagi, and S. Okada, Phys. Rev. B 81, 161403 (2010).

${ }^{22}$ J. Jung and A. H. MacDonald, arXiv:1208.0116.

${ }^{23}$ N. B. Kopnin, T. T. Heikkila, and G. E. Volovik, Phys. Rev. B 83, 220503(R) (2011); T. T. Heikkila and G. E. Volovik, JETP Lett. 93, 59 (2011); T. T. Heikkila, N. B. Kopnin, and G. E. Volovik, ibid. 94, 233 (2011); N. B. Kopnin and T. T. Heikkila, arXiv:1210.7075; N. B. Kopnin, M. Ijas, A. Harju, and T. T. Heikkila, arXiv:1210.7595.
${ }^{24}$ H. Liu, H. Jiang, X. C. Xie, and Q.-F. Sun, Phys. Rev. B 86, 085441 (2012).

${ }^{25}$ B. E. Feldman, J. Martin, and A. Yacoby, Nat. Phys. 5, 889 (2009).

${ }^{26}$ J. Martin, B. E. Feldman, R. T. Weitz, M. T. Allen, and A. Yacoby, Phys. Rev. Lett. 105, 256806 (2010).

${ }^{27}$ R. T. Weitz, M. T. Allen, B. E. Feldman, J. Martin, and A. Yacoby, Science 330, 812 (2010).

${ }^{28}$ A. S. Mayorov, D. C. Elias, M. Mucha-Kruczynski, R. V. Gorbachev, T. Tudorovskiy, A. Zhukov, S. V. Morozov, M. I. Katsnelson, V. I. Falko, A. K. Geim, and K. S. Novoselov, Science 333, 860 (2011).

${ }^{29}$ F. Freitag, J. Trbovic, M. Weiss, and C. Schonenberger, Phys. Rev. Lett. 108, 076602 (2012).

${ }^{30}$ J. Velasco, Jr., L. Jing, W. Bao, Y. Lee, P. Kratz, V. Aji, M. Bockrath, C. N. Lau, C. Varma, R. Stillwell, D. Smirnov, F. Zhang, J. Jung, and A. H. MacDonald, Nat. Nanotechnology 7, 156 (2012).

${ }^{31}$ A. Veligura, H. J. van Elferen, N. Tombros, J. C. Maan, U. Zeitler, and B. J. van Wees, Phys. Rev. B 85, 155412 (2012).

${ }^{32}$ M. F. Craciun, S. Russo, M. Yamamoto, J. B. Oostinga, A. F. Morpurgo, and S. Tarucha, Nat. Nanotechnology 4, 383 (2009).

${ }^{33}$ W. Bao, Z. Zhao, H. Zhang, G. Liu, P. Kratz, L. Jing, J. Velasco, Jr., D. Smirnov, and C. N. Lau, Phys. Rev. Lett. 105, 246601 (2010).

${ }^{34}$ W. Norimatsu and M. Kusunoki, Phys. Rev. B 81, 161410(R) (2010).

${ }^{35}$ K. F. Mak, J. Shan, and T. F. Heinz, Phys. Rev. Lett. 104, 176404 (2010).

${ }^{36}$ T. Taychatanapat, K. Watanabe, T. Taniguchi, and P. Jarillo-Herrero, Nat. Phys. 7, 621 (2011).

${ }^{37}$ A. Kumar, W. Escoffier, J. M. Poumirol, C. Faugeras, D. P. Arovas, M. M. Fogler, F. Guinea, S. Roche, M. Goiran, and B. Raquet, Phys. Rev. Lett. 107, 126806 (2011).

${ }^{38}$ L. Zhang, Y. Zhang, J. Camacho, M. Khodas, and I. Zaliznyak, Nat. Phys. 7, 953 (2011).

${ }^{39}$ C. H. Lui, Z. Q. Li, K. F. Mak, E. Cappelluti, and T. F. Heinz, Nat. Phys. 7, 944 (2011).

${ }^{40}$ Z. Li, C. H. Lui, E. Cappelluti, L. Benfatto, K. F. Mak, G. L. Carr, J. Shan, and T. F. Heinz, Phys. Rev. Lett. 108, 156801 (2012).

${ }^{41}$ W. Bao, L. Jing, J. Velasco Jr, Y. Lee, G. Liu, D. Tran, B. Standley, M. Aykol, S. B. Cronin, D. Smirnov, M. Koshino, E. McCann, M. Bockrath, and C. N. Lau, Nat. Phys. 7, 948 (2011).

${ }^{42}$ S. H. Jhang, M. F. Craciun, S. Schmidmeier, S. Tokumitsu, S. Russo, M. Yamamoto, Y. Skourski, J. Wosnitza, S. Tarucha, J. Eroms, and C. Strunk, Phys. Rev. B 84, 161408(R) (2011).

${ }^{43}$ Hao Wang and V. W. Scarola, Phys. Rev. B 83, 245109 (2011).

${ }^{44}$ Hao Wang and V. W. Scarola, Phys. Rev. B 85, 075438 (2012).

${ }^{45}$ M. S. Dresselhaus and G. Dresselhaus, Adv. Phys. 51, 1 (2002).

${ }^{46}$ S. Das Sarma, E. H. Hwang, and Wang-Kong Tse, Phys. Rev. B 75, 121406R (2007); S. Das Sarma, S. Adam, E. H. Hwang, and E. Rossi, Rev. Mod. Phys. 83, 407 (2011).

${ }^{47}$ EMSL Basis Set Exchange Library v1.2.2 at http://bse.pnl.gov/bse/.

${ }^{48}$ R. Wiesendanger, Rev. Mod. Phys. 81, 1495 (2009). 